\title{
Treinamento em transtornos mentais comuns na enfermaria: uso de metodologias ativas na construção do cuidado
}

\section{Marília Girão de Oliveira Machado \\ (D) https://orcid.org/0000-0001-7163-9061 \\ Cynthia Lima Sampaio ${ }^{2}$ \\ (D) https://orcid.org/0000-0003-0612-2781}

${ }^{1}$ Universidade Federal do Ceará, Fortaleza, CE, Brasil.

${ }^{2}$ Hospital Universitário Walter Cantídio, Fortaleza, CE, Brasil.
Objetivo: analisar a percepção de enfermeiras atuantes em leitos psiquiátricos de um hospital geral sobre a realização de um treinamento em saúde mental com a utilização de metodologias ativas. Método: pesquisa do tipo descritiva, exploratória, com abordagem qualitativa, realizada em um hospital geral de grande porte, com cinco enfermeiras, no período de junho a novembro de 2019. A coleta de dados consistiu em duas etapas: realização e avaliação do treinamento. Para a realização do treinamento, utilizaram-se metodologias ativas, com foco na aprendizagem baseada em problemas e problematização. A avaliação do treinamento foi realizada por meio de entrevista semiestruturada, elaborada segundo o modelo proposto por Kirkpatrick, e os dados analisados por meio da análise de conteúdo temática de Bardin. Resultados: os enfermeiros consideraram o treinamento positivo e desenvolveram habilidades como abordagem terapêutica, escuta ativa e mudança de comportamento. Conclusão: o treinamento foi eficaz na construção do conhecimento, promovendo transformações e reflexões significativas na aprendizagem de enfermeiros, além de contribuir com a produção de habilidades e atitudes na área de saúde mental, valorizando a aprendizagem significativa.

Descritores: Enfermagem Psiquiátrica; Saúde Mental; Aprendizagem Baseada em Problemas; Educação em Enfermagem.

\section{How to cite this article}

Machado MGO, Sampaio CL. Training on common mental disorders at a hospital ward: the use of active methodologies in care construction. SMAD, Rev Eletrônica Saúde Mental Álcool Drog. 2021 jan.- mar.;17(1):75-83. doi: https://dx.doi.org/10.11606/issn.1806-6976.smad.2021.168134 


\title{
Training on common mental disorders at a hospital ward: the use of active methodologies in care construction
}

\begin{abstract}
Objective: to analyze how nurses providing care to bedridden psychiatric patients in a general hospital perceive the conduction of mental health training by using active methodologies. Method: descriptive, exploratory, qualitative study including five nurses, carried out in a large general hospital from June to November 2019. Data collection consisted of two phases: performance and training evaluation. Active methodologies focused on problem-based learning and problematization were used to conduct the training. Training evaluation was performed by a semi-structured interview designed according to the model proposed by Kirkpatrick, and the data were analyzed by thematic content analysis according to Bardin. Results: the nurses considered the training to be positive and developed skills, such as therapeutic approach, active listening and behavior change. Conclusion: the training was effective in knowledge construction, thus promoting significant changes and reflections in nurses' learning, in addition to contributing to the development of skills and attitudes in mental health by valuing meaningful learning.
\end{abstract}

Descriptors: Psychiatric Nursing; Mental health; Problem-Based Learning; Nursing Education.

\section{Formación en trastornos mentales comunes en la enfermería: uso de metodologías activas en la construcción del cuidado}

Objetivo: analizar la percepción de las enfermeras que trabajan en las camas psiquiátricas de un hospital general sobre el desempeño de la formación en salud mental con el uso de metodologías activas. Método: investigación del tipo descriptiva, exploratoria, con un enfoque cualitativo, realizada en un gran hospital general con cinco enfermeras, de junio a noviembre de 2019. La recogida de datos consistió en dos etapas: la formación y su evaluación. Para llevar a cabo la formación se utilizaron metodologías activas, centradas en el aprendizaje basado en problemas y la problematización. La evaluación de la formación se realizó mediante una entrevista semiestructurada, elaborada según el modelo propuesto por Kirkpatrick, y los datos se analizaron mediante el análisis de contenido temático de Bardin. Resultados: las enfermeras consideraron positiva la formación y desarrollaron habilidades como el enfoque terapéutico, la escucha activa y el cambio de comportamiento. Conclusión: la formación fue eficaz en la creación de conocimientos, promoviendo transformaciones significativas y reflexiones en el aprendizaje de los enfermeros, además de contribuir a la producción de habilidades y actitudes en el área de la salud mental, valorando el aprendizaje significativo.

Descriptores: Enfermería Psiquiátrica; Salud Mental; Aprendizaje Basado en Problemas; Educación en Enfermería. 


\section{Introdução}

As metodologias ativas favorecem a autonomia dos profissionais de enfermagem, tornando-os sujeitos ativos e crítico-reflexivos, considerando a cultura, os saberes e a corresponsabilidade ${ }^{(1-2)}$. A equipe de enfermagem é estimulada a pensar, indagar e ressignificar seu aprendizado com o intuito de contribuir com a qualidade da assistência à saúde e romper os modelos tradicionais e hegemônicos nas práticas de saúde ${ }^{(3)}$.

A implementação do processo educativo com enfoque na Educação Permanente em Saúde (EPS) possibilita a aprendizagem contínua por meio da problematização da realidade, contribuindo, dessa forma, com o desenvolvimento de competências profissionais, de gestão e de controle social(4-5). Nesse sentido, a EPS busca repensar o desenvolvimento de competências técnicoassistenciais de enfermeiros, possibilitando a compreensão de princípios teórico-metodológicos e transformando profissionais de enfermagem ativos, preparados, críticos, integradores e éticos ${ }^{(2,4)}$.

Diante do exposto, para implementação da EPS, é necessário estabelecer comunicação com chefias das unidades de enfermarias, coordenação do setor de educação permanente e equipe de enfermagem, para incentivar o apoio institucional, por meio da adesão e participação de profissionais nas ações educativas. Essas estratégias requerem planejamento e disponibilidade para construção participativa de melhores resultados e comprometimento com o desenvolvimento permanente de recursos humanos ${ }^{(5)}$.

Nesse ínterim, as metodologias ativas são embasadas em duas abordagens problematizadoras: aprendizagem baseada em problemas, também conhecida como Problem Based Learning (PBL), e problematização. As duas propostas trabalham intencionalmente com problemas da realidade ${ }^{(6)}$.

O PBL utiliza problemas do cotidiano para promover o desenvolvimento de conceitos, procedimentos e atitudes no enfermeiro visando trabalhar de forma intencional os problemas da prática. Trata-se de uma metodologia formativa, pois busca estimular uma atitude ativa do aluno em busca do conhecimento(1,6). Difere-se, portanto, da problematização, em que os problemas são identificados pelo aluno, exercendo a cadeia dialética de ação - reflexão - ação, a partir da realidade observada, construindo uma aprendizagem dirigida e articulada(6-7).

Atualmente, no paradigma psicossocial, observa-se a necessidade de estimular e disseminar a capacidade pedagógica entre trabalhadores e gestores, de modo que ocorram mudanças positivas nas práticas profissionais. Destarte, um treinamento em saúde mental contribui com o aprimoramento de saberes e fazeres de enfermeiros, possibilitando o cuidado integral aos pacientes em sofrimento psíquico, além de instigar um processo educativo endógeno, contínuo e permanente ${ }^{(3,7-9)}$.

A carência de profissionais de enfermagem capacitados em saúde mental está relacionada a uma expansão dos serviços públicos especializados sem a oferta de capacitação, gerando indefinição nos papéis da equipe e sensação de nulidade ${ }^{(10)}$. Diante disso, destaca-se a utilização de metodologias de ensino reducionistas, fragmentadas e centradas no curativismo, em detrimento de práticas voltadas aos princípios e diretrizes do SUS. Tais modelos assistenciais hegemônicos descontextualizam o cotidiano do trabalho em saúde e dificultam a utilização de novos métodos de ensino(3,11).

Nessa realidade, o cuidado em saúde mental deve ser multidimensional e responder às necessidades do indivíduo, devendo dar conta da complexidade do ser em sofrimento psíquico, assim como importar reflexões e interações no contexto da formação de profissionais de enfermagem(3).

Com base nas considerações aqui delineadas, surgiu a seguinte questão norteadora: como enfermeiras analisam um treinamento com a utilização de metodologias ativas e como essas metodologias podem contribuir para o cuidado em saúde mental? O presente estudo teve como objetivo analisar a percepção de enfermeiras que atuam nos leitos psiquiátricos de um hospital geral sobre a realização de um treinamento em saúde mental com a utilização de metodologias ativas.

\section{Método}

Trata-se de um estudo exploratório-descritivo, com abordagem qualitativa, realizado na enfermaria da unidade de clínica médica que dispõe de leitos psiquiátricos, em um hospital geral de grande porte localizado na capital do Ceará, Brasil, no período de junho a novembro de 2019.

Fizeram parte da pesquisa enfermeiros assistenciais que atuam nos leitos psiquiátricos. O quadro atual de enfermeiros é composto por 18 profissionais. Os critérios de inclusão foram: ser enfermeiro com vínculo de, no mínimo, seis meses no serviço; fazer parte da enfermaria que dispõe de leitos psiquiátricos; e ter disponibilidade para participar em todos os momentos das etapas 01 e 02 da coleta de dados. Os critérios de exclusão consistiram em: estar de folga, férias e/ou licença e faltar em pelo menos um encontro da etapa 01.

A coleta de dados foi realizada em duas etapas. A etapa 01 consistiu na realização do treinamento. Foi entregue carta convite a todos os enfermeiros que se encaixavam nos critérios de inclusão e acordado, com a chefia da unidade, folga correspondente à carga horária de participação no treinamento para gozo de acordo com a disponibilidade do serviço. As atividades foram realizadas no horário de folga dos enfermeiros para que não houvesse prejuízo nas escalas de serviço. 
O treinamento ocorreu na sala de apoio didático do referido hospital, onde são destinadas as atividades de EPS, mediante agendamento prévio. Foi facilitado por enfermeiras do ambulatório de saúde mental/psiquiatria que compõem a equipe de pesquisa. Obteve carga horária total de 20 (vinte) horas divididas em quatro encontros semanais, sendo 16 horas presenciais e quatro horas de atividades de dispersão, estas elaboradas a partir de questões de aprendizagem implementadas em conjunto com as participantes ao final de cada treinamento. As respostas das questões de aprendizagem foram entregues e devidamente avaliadas pelos facilitadores no último dia de treinamento. As situações-problema foram criadas a partir de vivências na enfermaria que dispõe de leitos psiquiátricos do hospital e estudo de literaturas específicas de saúde mental e enfermagem.

O treinamento iniciou com a apresentação dos facilitadores, os objetivos, expectativas e a importância da presença dos participantes nos encontros subsequentes. Cada enfermeira recebeu um material impresso com uma situação-problema específica. As situações-problema foram denominadas como "Dilemas de Amanda", "Euforia de Larissa" e "Pensamentos de João" e buscaram abordar temas como cuidado ao paciente com anorexia, depressão, transtorno afetivo bipolar e esquizofrenia. Tratam-se de casos hipotéticos, com a utilização de nomes fictícios.

Utilizaram-se metodologias ativas com base no PBL e problematização por meio do Arco de Maguerez. Tratam-se de visões teóricas distintas, mas possuem pontos em comum(6). Ambas oportunizam a discussão sobre os transtornos mentais comuns na enfermaria a partir de problemas extraídos da realidade.

No treinamento, o PBL ocorreu durante as atividades de dispersão com a finalidade de fazer com que as enfermeiras estudassem determinados assuntos, estimulando, assim, uma atitude ativa em busca do conhecimento. As etapas do PBL consistem em: leitura do problema, identificação e esclarecimento de termos desconhecidos; identificação dos problemas propostos; formulação de hipóteses; resumo das hipóteses; formulação dos objetivos de aprendizado; estudo individual dos assuntos levantados nos objetivos de aprendizado; e rediscussão do problema ${ }^{(1,6)}$.

Paralelamente, na problematização, as facilitadoras conduziram o processo metodologicamente e as enfermeiras selecionaram os problemas para estudar, buscando respostas ou soluções para eles. Nesse sentido, o Arco de Maguerez inclui as seguintes fases: observação da realidade (problema); principais pontoschave; teorização; hipóteses de solução; e aplicação à realidade (prática) $)^{(6,12)}$.

A partir da leitura das situações-problema, os participantes falaram sobre suas vivências na enfermaria, o que sabiam a respeito das situações apresentadas, dúvidas sobre como abordar o paciente e dificuldades em promover a comunicação terapêutica, entre outras abordagens ao paciente em saúde mental, elencando os principais pontos-chave. Em seguida, a teorização foi implementada e discutida, gerando reflexões e adequando-se à prática na enfermaria. Por conseguinte, os participantes formularam hipóteses sobre o problema, elaborando questões de aprendizagem sobre os principais pontos-chave listados de acordo com a opinião de todos.

A etapa 2 consistiu na avaliação do treinamento. A coleta de dados ocorreu no próprio local de trabalho e foi realizada por meio da entrevista semiestruturada, com as seguintes questões norteadoras: 1 ) Comente sua avaliação referente ao treinamento em saúde mental; 2) Fale sobre que conhecimentos e/ou habilidades foram adquiridos nesse treinamento; 3) Explique como as discussões fomentadas durante o treinamento estão proporcionando ou não alguma mudança de comportamento na sua prática.

O referencial utilizado para fundamentar as questões norteadoras foi o Modelo de Avaliação, proposto por Kirkpatrick, que se fundamenta em quatro níveis de avaliação de treinamento (Figura 1). Esse referencial permite desenvolver a aprendizagem dentro do contexto da organização, por meio da avaliação e satisfação de profissionais em treinamentos, permitindo o desenvolvimento de formação permanente e qualidade nos serviços ${ }^{(13)}$. É composto pelos quatro seguintes níveis: referente ao treinamento, o conteúdo, o material $\begin{array}{cc}\text { Nível } 1 \text { - Reação } & \text { utilizado, o facilitador, a carga horária, entre } \\ \text { ou satisfação } & \text { outros. Tal medida pode proporcionar melhorias }\end{array}$ no treinamento, ou mesmo excluí-lo, se não for realizado de forma efetiva.

Permite avaliar princípios, fatos e técnicas observados e trazidos pelos participantes, ou

Nível 2 - seja, a aquisição de conhecimentos e habilidades Aprendizagem desses profissionais, como forma de perceber a realidade.

Avalia se os participantes obtiveram mudança de conduta ou comportamento com o treinamento.

Para que isso ocorra é preciso que a pessoa

Nível 3 Comportamento mudar, possua conhecimento para fazê-lo, busque auxílio na aplicação do aprendizado e, por último, seja premiada pela mudança.

Nível 4 Resultados Permite avaliar se os resultados foram alcançados e o seu impacto a partir da realização do treinamento.

Figura 1 - Níveis de Avaliação de Kirkpatrick. Fortaleza, Ceará, Brasil, 2019

Ressalta-se que a avaliação do nível 4 de Kirkpatrick não foi realizada nesse momento, visto que a avaliação dos resultados requer mensuração do impacto na assistência, o que envolve a identificação de indicadores e acompanhamento em médio e longo prazo. Portanto, serão necessários estudos posteriores para avaliar esse nível.

As falas das participantes foram gravadas e transcritas na íntegra pelo próprio entrevistador. Os dados 
foram distribuídos por unidades de análise temática e posteriormente categorizadas para proceder-se à análise e discussão dos resultados. Utilizou-se a análise de conteúdo temática, a partir das seguintes etapas: pré-análise, exploração do material e interpretação dos resultados ${ }^{(14)}$. O estudo foi submetido e aprovado pelo Comitê de Ética e Pesquisa (CEP), sob o protocolo 3.464.527, em 22 de julho de 2019, atendendo à Resolução 466/12 do Conselho Nacional de Saúde(15).

\section{Resultados}

Participaram do estudo cinco enfermeiras. Todas pertencem ao sexo feminino, com idade variando entre 31 e 40 anos. Destas, três possuem cursos de pós-graduação do tipo lato sensu, uma realizou mestrado e uma está cursando o doutorado. Quanto ao regime de contrato de trabalho na instituição, quatro trabalham pelo regime de Consolidação das Leis do Trabalho (CLT), enquanto uma trabalha por Regime Jurídico Único (RJU). Com relação ao tempo de atuação na instituição, duas enfermeiras estão no serviço entre 1-2 anos, duas estão entre 2-5 anos e uma $>5$ anos.

Das falas das entrevistadas emergiram três categorias temáticas: avaliação da reação ou satisfação; avaliação da aprendizagem; e avaliação do comportamento.

\section{Avaliação da reação ou satisfação}

Evidenciou-se, por meio das falas, que o treinamento foi considerado positivo e que o uso de metodologias ativas proporcionou melhorias na assistência de enfermagem em saúde mental. Utilizaram-se estratégias de ensino contextualizadas e participativas sobre situações recorrentes na prática e na diversidade de demandas dos leitos psiquiátricos, promovendo ampla discussão e reflexão sobre aspectos teóricos e práticos com foco na realidade vivenciada. A minha avaliação sobre o treinamento e utilização de metodologias ativas é uma avaliação bem positiva. Foi possível trocar conhecimentos, aprender, também muitas coisas novas sobre os principais transtornos mentais que acometem os pacientes internados. Foi algo muito voltado para nossa prática profissional que contribuiu muito com a nossa assistência, foi muito positivo a forma como o curso implementado, por meio de uma metodologia ativa que propiciou essa troca de conhecimento e uma aprendizagem bem significativa (E2).

No entanto, algumas enfermeiras pontuaram que o treinamento poderia ser ofertado mais vezes e que fossem abordados mais assuntos em saúde mental. É importante frisar que o treinamento foi uma necessidade das próprias participantes e da instituição, com vistas a ampliar/extrapolar o conhecimento técnico-científico e promover resultados positivos no dia a dia dos serviços. Eu gostei bastante, como eu tinha falado aqui na minha avaliação escrita, eu queria que tivesse mais tempo e que a gente abordasse mais assuntos? Mas eu achei superválido... abriu minha mente também para um relacionamento terapêutico melhor com esses pacientes com transtornos mentais... e melhorar também a minha postura como enfermeira no cuidado com eles... então eu gostei realmente do curso e queria que tivesse só um pouco mais de horas (E5).

Nota-se que a satisfação das enfermeiras perante o treinamento remete a várias pactuações e levantamentos de soluções para repensar estratégias de cuidado por meio da troca de experiências e protagonismo da participação. Todas as participantes trabalharam simultaneamente na utilização do PBL e na metodologia da problematização, o que reforça o processo de autonomia. Isso favorece a criação de caminhos possíveis para o desenvolvimento de respostas ou soluções para os problemas que se apresentam.

\section{Avaliação da aprendizagem}

Tendo em vista que o conhecimento construído de forma colaborativa auxilia na compreensão de fatos que se aproximam com a realidade, o uso de metodologias ativas tornou-se estratégico para obtenção de conhecimento em saúde mental, pois implicou na construção de habilidades de comunicação e capacidade de raciocínio crítico, minimizando, assim, as dificuldades e tornando o profissional mais preparado para lidar com certas circunstâncias.

Eu tinha muita dúvida em relação a como abordar o paciente com esquizofrenia, transtorno bipolar, enfim, eu acho que foi para mim a parte mais eficaz em questão da comunicação. O que eu devo falar, o que eu não devo. O que eu devo observar, né? Eu acho que foi mais as habilidades de comunicação que eu desenvolvi mais... a questão do tratamento, do cuidado ao paciente (E1).

Algumas enfermeiras revelaram dúvidas a respeito dos transtornos psiquiátricos abordados nas situaçõesproblema, tais como sintomas, tratamentos e condutas de enfermagem diante de cada caso. Cumpre ressaltar que os diagnósticos e intervenções de enfermagem foram trazidos no final de cada situação a fim de oferecer contributos importantes para a prática e ampliar as possibilidades de cuidado, conforme citou E4: ...os principais sintomas apresentados nos transtornos, os principais elementos, as características dos transtornos... isso é muito importante porque permite que a gente consiga identificar... esses elementos no paciente que a gente está cuidando e principalmente como conduzir... que foi algo que a gente falou muito... sobre como conduzir... Os sintomas apresentados nos principais transtornos... foram conhecimentos muito importantes... principalmente os diagnósticos de enfermagem e as condutas a serem implementadas em cada caso (E4).

Os saberes prévios alinhados às situações problematizadoras abordadas no treinamento favoreceram a aquisição de conhecimentos e habilidades de cuidados 
por parte das entrevistadas, tornando as atividades de EPS mais presentes no cotidiano do trabalho. A aprendizagem tornou-se dinâmica, visto que permitiu a interação entre os diversos atores, ao mesmo tempo que as enfermeiras ficaram motivadas a aprender. Para mim, a habilidade que eu posso até desenvolver mais é a questão da abordagem terapêutica. De como chegar mais no paciente e na família. Eu acho que ficou mais tranquilo essa parte. Tanto conhecimento quanto as habilidades (E5).

À medida que os encontros ocorriam, cada participante começava a ter clareza dos objetivos e natureza das metodologias ativas, com avanço significativo nas discussões referentes às situações-problema. A busca pela construção de significados, de forma coletiva, foi uma ferramenta útil para que as participantes se tornassem questionadoras sobre como o cuidado em saúde mental deve ser ofertado na enfermaria. Nesse sentido, o treinamento possibilitou uma conotação mais dinâmica no processo ensino-aprendizagem, oportunizando a compreensão mais profunda do cuidado e a estimulação do pensamento crítico dentro do contexto biopsicossocial.

\section{Avaliação do comportamento}

A reflexão coletiva sobre o cuidado em saúde mental esteve presente na maioria das falas. Nota-se que houve mudança de comportamento durante e depois do treinamento e que o mesmo proporcionou novos caminhos sobre o processo do cuidado, tornando-se mais humanizado e integralizado. Houve uma mudança de visão mesmo. Hoje eu não entro mais para atender um paciente como eu entrava antes do treinamento. Eu acho que vocês fomentaram muito essa questão de olhar com empatia, de falar coisas que estimulem o paciente a falar. Você falou muito dos pacientes com delírios e alucinações, de trazer eles sempre para a realidade. Procurar sempre fazer uma abordagem terapêutica com eles antes de física ou química, que é o que a gente na maioria das vezes fazia. Paciente em crise a gente sempre questiona... qual é o remédio que eu vou dar? E assim vocês buscaram uma visão, uma abordagem terapêutica sem tratar necessariamente da química ou da mecânica. Se caso essas não fossem tão eficazes, aí ia para outras alternativas. Eu acho que as discussões também foram fundamentais para mudar meu comportamento... da abordagem ao paciente (E1).

Observou-se que a utilização de metodologias ativas atuou na (re)construção de saberes em saúde mental e que trabalhar de forma empática buscou superar o modelo biológico-tecnicista, muito comum em locais onde se ofertam cuidados em saúde mental. Nessa perspectiva ampliada de cuidado, apontam-se caminhos para mudanças de condutas e redirecionamentos em saúde mental. É algo que ainda está acontecendo, mas eu já percebi isso. Eu já parei um pouco para refletir como que eu posso melhorar o meu cuidado, a minha conduta, a minha forma de abordagem... eu percebi que muitas vezes eu estava abordando errado, então, isso já é algo positivo. É muito conhecimento... e o curso na verdade serviu também para nos estimular a aprender mais, a buscar mais conhecimento, a estudar mais... assim, eu me sinto instigada a procurar mais conhecimento, a aprofundar mais em relação a esse cuidado ao paciente com transtorno mental (E2).

Destarte, verifica-se que as enfermeiras aprenderam a identificar as suas próprias necessidades de aprendizado, levando em consideração conhecimentos e experiências prévias. Desde o primeiro encontro... quando a gente saiu daqui e aí a gente chegou novamente na enfermaria, eu acho que até a abordagem, o entrar, o mostrar mais participativo, a questão mesmo de chegar, de perguntar, mas de uma maneira bem ampliada. Então essa abordagem que eu não tinha antes, hoje eu já tenho desde o primeiro encontro. Hoje já existe um certo cuidado maior, da assistência, tanto clínica quanto psíquica... de dar um apoio mesmo àquela família, a gente já tem um cuidado maior, não que a gente não tivesse antes, mas hoje a gente tem mais zelo... eu acho que eu diria isso. Então eu vejo melhora mesmo na minha prática (E3).

Diante das construções formuladas no treinamento com a utilização de metodologias ativas, a mudança de comportamento favoreceu transformações significativas no planejar e agir durante a abordagem aos pacientes psiquiátricos. Os dados encontrados revelaram que as enfermeiras avançaram no desenvolvimento de habilidades de pensamento crítico e criativo. Apesar das dificuldades iniciais, elas analisaram positivamente o treinamento.

\section{Discussão}

A satisfação das enfermeiras, evidenciada na avaliação do treinamento, ocorreu por meio da inter-relação teoria e prática, contribuindo com o exercício da práxis educativa. A vivência de diferentes caminhos metodológicos permitiu-Ihes o envolvimento com aspectos da realidade e meios para sua superação. Assim sendo, as enfermeiras apontaram alternativas para o cuidado ao paciente em sofrimento mental, dada a sua importância na relação terapêutica e transformação das práticas de saúde ${ }^{(3,16)}$.

Nas metodologias ativas utilizadas no treinamento em transtornos mentais, as enfermeiras ocuparam o centro das ações educativas e construíram o conhecimento de forma participativa. Faz-se oportuno frisar que a EPS e o método tradicional de ensino são discordantes. Nesse último, os discentes ocupam uma posição de passividade e não possuem uma participação de forma mútua. A educação problematizadora, nesse sentido, permite que o aprendiz participe ativamente da tomada de decisões, sendo o educador o mediador desse processo(2,4,17).

No contexto internacional, a educação em saúde mental tem se mostrado satisfatória, na medida em que favorece a competência e prontidão para o gerenciamento de casos. Estudo realizado em Hong Kong sobre treinamento em saúde mental mostrou-se 
eficaz para reforçar conhecimentos, aprimorar técnicas e habilidades, além de propiciar um maior senso de conquista e satisfação no aprendizado de estudantes de enfermagem(18). Verifica-se, portanto, que atividades educativas em saúde mental podem tornar o cuidado de enfermagem mais resolutivo e garantir a construção de práticas pautadas em realidades vivas.

No entanto, outros estudos nacionais e internacionais revelam que a maioria dos treinamentos em saúde mental está embasada em uma lógica formativa tradicional, além da pouca oferta de cursos de qualificação e aperfeiçoamento, carga horária teórico-prática insuficiente e as próprias limitações impostas pelo modelo biomédico. Aliado a essas inquietações, é notório destacar que o estigma social da doença mental é um fator determinante que pode, consequentemente, ser gerador de sobrecarga em enfermeiros, devendo ser elaborado e repensado durante as atividades de formação permanente ${ }^{(8,11,16)}$.

No que tange às ferramentas de cuidado, as enfermeiras identificaram a importância da comunicação terapêutica como abordagem central, elencando a escuta ativa preponderante ao aconselhamento, muitas vezes, identificada como uma necessidade do próprio profissional. A comunicação terapêutica é uma tecnologia leve e exige técnica, como ouvir reflexivamente e observar a linguagem verbal e não verbal do paciente, além de enriquecer o relacionamento com a equipe multidisciplinar. Assim, o cuidado deve abordar a informação, educação e apoio ao paciente em seu processo saúde-doença, de forma a proporcionar um plano terapêutico eficaz visando um atendimento integral por meio da manutenção do funcionamento psicossocial(16,19-22).

As enfermeiras do estudo analisaram o treinamento como positivo, pois, conforme trouxeram nos resultados, os elementos problematizadores do treinamento favoreceram a mudança de comportamentos, habilidades e atitudes por meio do exercício de atividades centradas em papéis terapêuticos(23). Nesse sentido, as enfermeiras compreenderam que processos ativos e dinâmicos de aprendizagem fornecem o ambiente ideal para uma assistência de enfermagem qualificada e capaz de atuar resolutamente nos mais diversos contextos em saúde mental.

Destaca-se, como um importante componente do cuidado em saúde mental na contemporaneidade, a formação que induza profissionais à valorização de fazeres mais críticos com a identificação de necessidades subjetivas e sociais dos pacientes em sofrimento mental(24). As práticas de cuidado humanizado, por sua vez, favorecem o vínculo entre a equipe de enfermagem e o usuário em sua totalidade, promovendo uma intervenção ampliada por meio da substituição do termo "tratar" por "cuidar"(10,25).

Logo, o cuidado deve oferecer uma perspectiva holística e ir além da visão reducionista à doença. A dimensão emocional do cuidado de enfermagem vai além do corpo, portanto, reconhece-se a necessidade de formação de profissionais com base em uma práxis pedagógica crítica e humanista, a partir de habilidades de relacionamento interpessoal(26-27).

O relacionamento interpessoal baseia-se na construção de vínculo, segurança e humanização, tendo por objetivo tornar o paciente ativo em sua produção de vida. Tanto o enfermeiro quanto o paciente buscam juntos melhores soluções para o restabelecimento da saúde, seja ela física ou psíquica. Por isso, requer a construção relacional conjunta e dialógica, com vistas a proporcionar um cuidado adequado e efetivo para os principais problemas em saúde mental(26-27). Isso foi alcançado por meio do treinamento, uma vez que as enfermeiras participaram de modo integrado e afetivo, desenvolvendo pensamentos, sentimentos e ações necessárias ao cuidado em saúde mental.

Durante as discussões no treinamento, foi possível lançar luz sobre aspectos relacionados à abordagem familiar. Esta, segundo a lógica psicossocial, é entendida como parte fundamental para a evolução satisfatória do paciente e precisa ser multidimensional, devendo a equipe de enfermagem incentivar e integrar a participação da família no tratamento e reabilitação de pacientes com transtornos mentais. O enfermeiro, portanto, deve acolher as diferentes constituições familiares, atuando com respeito aos sentimentos e emoções presentes no sofrimento psíquico, a fim de estimular o processo do enfrentamento das dificuldades ${ }^{(16,28)}$.

Desse modo, com a utilização de metodologias ativas, as enfermeiras foram confrontadas com as situações da vida real, corroborando uma melhor análise dos problemas rotineiros com consequente busca de soluções, transcendendo a prática profissional. Colocar as enfermeiras diante de problemas e desafios foi essencial para mobilização de seus potenciais intelectuais. Logo, ressalta-se a importância do raciocínio clínico como competência básica para aquisição de conhecimentos e incentivo à tomada de decisões, visto que as participantes foram motivadas a buscar novas informações a fim de garantir um aprendizado autodirigido e colaborativo ${ }^{(1,17)}$.

Conclui-se que as enfermeiras analisaram o treinamento de forma positiva e ficaram satisfeitas com as metodologias utilizadas, pois tornaram-se capazes de se deter, examinar e ressignificar suas descobertas e aprendizados em saúde mental. A aprendizagem significativa viabilizou mudanças na prática e nas percepções de enfermeiros, bem como potencializou a construção de ações pautadas dentro dos pressupostos da reforma psiquiátrica e dos princípios e diretrizes do Sistema Único de Saúde (SUS).

Este estudo contribuiu para a produção de conhecimento na área de saúde mental com a utilização 
de metodologias ativas de ensino por meio de um treinamento em transtornos mentais. Esse último buscou ampliar conhecimentos, habilidades e atitudes, além da capacidade crítica no exercício cotidiano do trabalho. Considera-se como limitação do estudo a dificuldade de disponibilidade e adesão de enfermeiros para participarem do treinamento. Sugere-se que mais treinamentos como esse sejam aperfeiçoados e implementados em articulação com o cenário psicossocial.

\section{Conclusão}

Pode-se concluir que as enfermeiras analisaram o treinamento e a utilização de metodologias ativas como essenciais e estratégicos para a promoção de mudanças democráticas e integradoras no cuidado em saúde mental, demandando mais que conhecimentos técnicos e científicos, mas habilidades para saber lidar com comportamentos e relações humanas.

Evidenciou-se que o treinamento se configurou como uma experiência exitosa, uma vez que impulsionou enfermeiros a construírem novos caminhos para o cuidado, buscando a melhoria contínua da prática de enfermagem em saúde mental.

Ressalta-se a importância de mais treinamentos em saúde mental com a utilização de metodologias ativas de ensino, de modo a atuar na construção de espaços coletivos para reflexão das ações no cotidiano dos serviços de saúde. Espera-se que este estudo estimule a continuidade de novos treinamentos e colabore com a formação e inovação de práticas de cuidado em saúde mental.

\section{Referências}

1. Arrue M, Alegría BR, Zarandona J, Cillero IH. Effect of a PBL teaching method on learning about nursing care for patients with depression. Nurse Educ Today. May 2017;52: 109-15. doi: https://doi.org/10.1016/j.nedt.2017.02.016 2. Fernandes MA, Soares NSA, Ribeiro IAP, Sousa CCM, Ribeiro HKP. Active methodologies as a tool for training in mental health. Rev Enferm UFPE on line. 2018 dez;12(12):3172-80.doi: https://doi.org/10.5205/19818963-v12i12a237762p3172-3180-2018

3. Cruz RAO, Araujo ELM, Nascimento NM, Lima RJ, França JRFS, Oliveira JS. Reflections in the light of the complexity theory and nursing education. Rev Bras Enferm.[Internet]. 2017;70(1):224-7. doi: http://dx.doi. org/10.1590/0034-7167-2016-0239

4. Gigante RL, Campos GWS. Continuing training and education in health policy in Brazil: legal basis and theoretical referencess. Trabalho, Educação e Saúde. 2016;14(3):747-63. doi: https://doi. org/10.1590/1981-7746-sip00124

5. Sade PMCS, Peres AM, Brusamarelo T, Mercês NNA, Wolff LDG, Lowen IMV. Continuous nursing education requirements in a teaching hospital. Cogitare Enferm. 2019; 24. doi: http://dx.doi.org/10.5380/ce.v24i0.57130 6. Berbel NAN. "Problematization" and problem-based learning: different words or different ways? Interface. (Botucatu). 1998;2(2):139-54. doi: https://doi. org/10.1590/S1414-32831998000100008

7. Leal LB, Pereira KLA, Negreiros ALB, Pequeno AMC, Lima GC, Negreiros FDS, et al. Active problematizing method as a strategy for training in health Rev Enferm UFPE on-line.[Internet]. 2018;12(4):1139-43. doi: https://doi. org/10.5205/1981-8963-v12i4a231346p1139-1143-2018 8. Pessoa JM Júnior, Santos RCA, Clementino FS, Nascimento EGC, Miranda FAN. Mental health education and professional practice in the psychiatric hospital. Texto Contexto Enferm, 2016;25(3):e3020015. doi: https://doi. org/10.1590/0104-07072016003020015.

9. Rézio LA, Fortuna CM, Borges FA. Tips for permanent education in mental health in primary care guided by the Institutional Socio-clinic. Rev. Latino-Am. Enfermagem. 2019;27:e3204. doi:https://doi. org/10.1590/1518-8345.3217.3204

10. Braga FS, Olschowsky. A Pleasure and suffering in the work of mental health nurses in the context of the psychiatric reform. Rev Enferm UFPE online. [Internet]. 2015;9(3):7086-94. Available from: http://www.lume. ufrgs.br/bitstream/handle/10183/115332/000956660. pdf?sequence $=1$

11. Sobral F, Campos C. Nurses and mental health education in primary care: an integrative review. SMAD Rev Eletr Saúde Mental Álcool Drog [Internet]. 1 ago.2012;8(2):100-7. doi: https://doi.org/10.11606/ issn.1806-6976.v8i2p100-107

12. Bordenave JD, Pereira AM. Estratégias de ensinoaprendizagem. 25a ed. Rio de Janeiro: Vozes; 2004.

13. Kirkpatrick DL, Kirkpatrick JD. Como avaliar programas de treinamento de equipes - os quatro níveis. Rio de Janeiro: SENAC-RIO; 2010.

14. Bardin L. Análise de conteúdo. São Paulo: Edições 70; 2011.

15. Ministério da Saúde (BR). Conselho Nacional de Saúde. Resolução n. 466, de 12 de dezembro de 2012. Aprova as diretrizes e normas regulamentadoras de pesquisas envolvendo seres humanos. Diário Oficial da União. Brasília (DF); 2013 jun 13; Seção 1, p. 59-62.

16. Mota AS, Silva ALA, Souza AC. Educação permanente: Ongoing education: Practices and processes related to mental health nursing. Rev Portuguesa Enferm Saúde Mental. [Internet]. 2016;(spe4):9-16. doi: http://dx.doi. org/10.19131/rpesm.0135

17. Wosinski J, Belcher AE, Dürrenberger $Y$, Allin AC, Stormacq C, Gerson L. Facilitating problem-based learning among undergraduate nursing students: A qualitative systematic review. Nurse Educ Today. 2018;(60):67-74. doi: http://dx.doi.org/10.1016/j.nedt.2017.08.015. 
18. Hung MSY, Lam SKK, Chow MCM. Nursing students' experiences of mental health first aid training: A qualitative descriptive study. Collegian. 2019;26 (5):534-40.doi: https://doi.org/10.1016/j.colegn.2019.02.006

19. Furnes M, Kvaal KS, Høye S Communication in mental health nursing - Bachelor Students' appraisal of a blended learning training programme - an exploratory study. BMC Nurs. 2018;17(20):1-10. doi: https://doi.org/10.1186/ s12912-018-0288-9

20. Nascimento MGG, Nadaleti NP, Nadaleti SC, Vilela SC, Terra FS, Silva SA, Resck ZMR. nursing work process in the promotion of mental health: reflective analysis. RECOM, Rev Enferm Centro Oeste Mineiro. 2017;7:e2097. doi: http://dx.doi.org/10.19175/recom.v7i0.2097

21. Beurs, DP, Groot MH, Keijser J, Dujin EV, Winter RFP, Kerkhof FM. Evaluation of benefit to patients of training mental health professionals in suicide guidelines: cluster randomised trial. $\mathrm{Br}$ J Psychiatry. [Internet]. 2016;208(5):477-83. doi: https://doi.org/10.1192/bjp. bp. 114.156208

22. Lima FFL, Alencar NES, Fernandes MA, Moura ECCM, Silva-Júnior FJG.. Situational strategic planning in a psychiatric institution: contributions and challenges. SMAD Rev Eletr Saúde Mental Álcool Drog [Internet]. 28 ago.2019;15(2):20-8. Available from: 10.11606/ issn.1806-6976.smad.2019.000377

23. Gould BH, Brodie L, Carver F, Logan P. Not just ticking all the boxes. Problem based learning and mental health nursing. A review. Nurse Educ Today. 2015;35(10):e1-e5. doi:http://dx.doi.org/10.1016/j.nedt.2015.07.013

24. Luis MAV. Current knowledge demands in mental health care. SMAD Rev Eletr Saúde Mental Álcool Drog [Internet]. 31mar.2018;14(1):1-. doi: http://dx.doi. org/10.11606/issn.1806-6976.smad.2018.152779

25. Canabarro JL. Inserção de residentes multiprofissionais nos serviços de saúde mental: percepção dos trabalhadores. Dissertação (Mestrado em enfermagem). Santa Maria: Universidade Federal de Santa Maria; 2017. Available from:https://repositorio.ufsm.br/bitstream/ handle/1/11818/Canabarro\%2C\%20Jana\%C3\%ADna\%20 Lunardi. pdf?sequence $=1$ \&isAllowed $=y$

26. Monteiro PV, Almeida ANS, Pereira MLD, Freitas MC, Guedes MVC, Silva LF. When body care is not enough: the emotional dimension of nursing care. REME - Rev Min Enferm. 2016;20: e957.doi: http://dx.doi. org/10.5935/1415-2762.20160026

27. Fernandes MA, Almeida JS, Oliveira EKC, Sousa $\mathrm{KHJF}$. Nursing process based on Peplau interpersonal relationship theory applied to schizophrenia. Rev Enferm UFPI. 2018 Jul-Sep;7(3):42-7. doi: http://dx.doi. org/10.26694/2238-7234.7342-47

28. Buriola AA, Kantorski LP, Sales CA, Matsuda LM. Nursing practice at a psychiatric emergency service: evaluation using fourth generation assessment. Texto Contexto Enferm, 2016; 25(1):e4540014. Available from: https://doi.org/10.1590/0104-070720160004540014

\section{Contribuição dos Autores}

Concepção e planejamento do estudo: Marília Girão de Oliveira Machado e Cynthia Lima Sampaio. Obtenção dos dados: Marília Girão de Oliveira Machado e Cynthia Lima Sampaio. Análise e interpretação dos dados: Marília Girão de Oliveira Machado e Cynthia Lima Sampaio. Redação do manuscrito: Marília Girão de Oliveira Machado. Revisão crítica do manuscrito: Marília Girão de Oliveira Machado.

Todos os autores aprovaram a versão final do texto. Conflito de interesse: os autores declararam que não há conflito de interesse.
Copyright $\odot 2021$ SMAD, Rev. Eletrônica Saúde Mental Álcool Drog. Este é um artigo de acesso aberto distribuído sob os termos da Licença Creative Commons CC BY-NC.

Esta licença permite que outros remixem, adaptem e criem a partir do seu trabalho para fins não comerciais, e embora os novos trabalhos tenham de lhe atribuir o devido crédito e não possam ser usados para fins comerciais, os usuários não têm de licenciar esses trabalhos derivados sob os mesmos termos. 